Herz 2019 • 44:573

https://doi.org/10.1007/s00059-019-04858-2

(c) Springer Medizin Verlag GmbH, ein Teil von Springer Nature 2019

\title{
R. Zahn
}

Medizinische Klinik B, Klinikum der Stadt Ludwigshafen am Rhein gGmbH, Ludwigshafen, Deutschland

\section{Interventionelle Therapie der Mitralklappe - ein Update}

Die interventionelle Therapie der Herzklappen ist eines der spannendsten kardiologischen Themen der letzten Jahre. Nachdem die Transkatheteraortenklappenimplantation (TAVI) die Therapie der symptomatischen Aortenklappenstenose revolutioniert hat und mittlerweile für die meisten Patienten zur Methode der Wahl geworden ist, geht es mit der interventionellen Therapie der Mitralklappenfehler, insbesondere der Mitralinsuffizienz, deutlich langsamer voran. Das vorliegende Heft von Herz soll hier ein Update über den aktuellen Stand von Diagnostik und Therapie geben.

Im Artikel von Kreidel wird der aktuelle Stand der echokardiographischen Diagnostik der Mitralinsuffizienz dargestellt. Anders als bei der Aortenstenose, wo es klare Parameter zur Schweregradbeurteilung gibt, ist es bei der Mitralinsuffizienz eher ein Bausteinprinzip, wo unterschiedliche Parameter zu einer Gesamtbeurteilung integriert werden müssen.

Boekstegers stellt die Entwicklung der Edge-to-edge-Therapie seit dem Positionspapier der Deutschen Gesellschaft für Kardiologie 2013 dar.

Friedrichs und Rudolf versuchen, die unterschiedlichen Ergebnisse der beiden großen randomisierten Studien zur Therapie der sekundären Mitralinsuffizienz mittels MitraClip ${ }^{\circledR}$, MITRA-FR und COAPT, zu erklären.

Frerker et al. stellen die aktuellen Daten zur Therapie mit dem Cardioband ${ }^{\circledR}$ vor und versuchen, die doch eingeschränkten Indikationen zu erklären.

Im letzten Artikel geben Vogelhuber et al. einen Überblick über den aktuellen Stand der Entwicklung der interventionellen Mitralklappen.
Wir hoffen, Sie mit dem vorliegenden Heft auf den aktuellen Informationsstand bezüglich eines sich rasch entwickelnden, spannenden Themas zu bringen.

\section{R. Zahn}

\section{Korrespondenzadresse}

Prof. Dr. med. R. Zahn

Medizinische Klinik B, Klinikum der Stadt Ludwigshafen am Rhein $\mathrm{gGmbH}$ Bremserstraße 79, 67063 Ludwigshafen, Deutschland

erzahn@aol.com

Interessenkonflikt. R. Zahn gibt an, dass kein Interessenkonflikt besteht 